\title{
Current Perspectives on HER2 Testing: A Review of National Testing Guidelines
}

\author{
Michael Bilous, M.A., M.B., Ch.B., F.R.C.P.A., Mitch Dowsett, Ph.D., Wedad Hanna, M.D., \\ Jorma Isola, M.D., Ph.D., Annette Lebeau, M.D., Aberlardo Moreno, M.D., \\ Frédérique Penault-Llorca, M.D., Ph.D., Josef Rüschoff, M.D., Gorana Tomasic, M.D. \\ Marc van de Vijver, M.D., Ph.D.
}

Institute of Clinical Pathology and Medical Research (MB), Westmead Hospital, Westmead, New South Wales, Australia; The Royal Marsden NHS Trust (MD), London, United Kingdom; Sunnybrook \& Women's College Health Science Center (WH), University of Toronto, Toronto, Canada; University of Tampere (JI), Tampere, Finland; Pathologisches Institut der LMU Muenchen (AL), Muenchen, Germany; Ciutat Santatia de Bellvitge (AM), Barcelona, Spain; Centre Jean Perrin (FPL), Clermont-Ferrand, France; Institut für Pathologie (JR), Kassel, Germany; Instituto Nazionale dei Tumori (GT), Milano, Italy; The Netherlands Cancer Institute (MvdV), Amsterdam, The Netherlands

\begin{abstract}
Knowledge of HER2 status is a prerequisite when considering a patient's eligibility for Herceptin (trastuzumab) therapy. Accurate assessment of HER2 status is essential to ensure that all patients who may benefit from Herceptin are correctly identified. There are several assays available to determine HER2 status: the most common in routine clinical practice are immunohistochemistry (IHC) and fluorescence in situ hybridization (FISH). Various factors can affect the results achieved with these assays, including the assay antibody/probe, the methodology and the experience of personnel. Many countries have implemented national testing guidelines in an attempt to standardize testing procedures and make results more accurate. These guidelines vary in the level of detail and the number of recommendations. This review looks at areas of consensus between the different national testing guidelines and highlights where errors may arise during the testing procedure. The key point underlined by this review is that whatever method is used to test for HER2 status, the technology must be validated first, and there must be regular internal and external quality control and quality assurance procedures.
\end{abstract}

\footnotetext{
Copyright (C) 2003 by The United States and Canadian Academy of Pathology, Inc.

VOL. 16, NO. 2, P. 173, 2003 Printed in the U.S.A

Date of acceptance: November 8, 2002.

Authors are listed alphabetically.

Supported by an educational grant from F. Hoffmann-La Roche.

Address reprint requests to: Wedad Hanna, M.D., Sunnybrook and Women's College Health Sciences Centre, Department of Anatomic Pathology,

E4-36, 2075 Bayview Avenue, Toronto, ON M4N 3M5, Canada; e-mail: wedad.hanna@swchsc.on.ca.

DOI: 10.1097/01.MP.0000052102.90815.82
}

KEY WORDS: Breast cancer, Fluorescence in situ hybridization, HER2, Immunohistochemistry, Testing guidelines.

Mod Pathol 2003;16(2):173-182

Progress in molecular biology has resulted in the identification and greater understanding of molecular markers that may have prognostic and predictive value for breast cancer patients. The human epidermal growth factor receptor-2 (HER2/neu/cerbB-2) is one of the best characterized of such markers. The subset of patients with breast cancer demonstrating a HER2-positive status has aggressive tumors and a poor prognosis (1-3). There is mounting evidence that HER2 status may predict response to chemotherapy and hormonal therapy, although conclusive data are needed $(4,5)$. Most important, demonstration of high HER2 receptor overexpression or HER2 gene amplification is essential for treatment with the anti-HER2 monoclonal antibody therapy Herceptin, which has significant clinical benefits in patients with metastatic breast cancer (6-8). Clinical studies have also shown that the level of HER2 overexpression correlates with clinical benefit. Patients whose tumors have high HER2 receptor overexpression and/or amplification of the HER2 gene benefit most from Herceptin (7, 9-11). For these reasons, testing for HER2 status is important for the management of patients with breast cancer, and accurately assessing HER2 status is essential in deciding which patients will benefit from Herceptin therapy.

Currently, no single assay is globally accepted as the gold standard for HER2 testing. Factors that can lead to inaccuracies in HER2 testing results include preparation, fixation, and storage of the tissue sec- 
tions; the antibody or probe used to detect HER2; scoring or result interpretation; lack of validation of methodologies; experience of personnel; and interobserver variability.

Given the wide variation in testing procedures it is difficult to suggest gold-standard HER2 testing guidelines. As a first step toward standardizing HER2 testing procedures at a local level, several countries have developed national guidelines for diagnostic centers to follow. These include published guidelines from Australia (12), Canada (13), France (14), Germany (15), Japan (16), and the United Kingdom (17) and unpublished guidelines from the Czech Republic, Finland, Sweden, and The Netherlands, which are used routinely. Recommendations by the American Society of Clinical Oncology (ASCO; 18) and by the College of American Pathologists (CAP; 19) have also been published. The national guidelines vary in the actual recommendations and the level of detail (Table 1). The guidelines are continuously evolving as we better understand the issues surrounding HER2 testing and more data on HER2 and response to Herceptin become available. This review summarizes the areas of agreement in the current national testing guidelines and highlights issues in HER2 testing that can lead to variable results. By giving an overview of the national testing guidelines, we hope that areas of agreement and disagreement can be identified and considered. This should lead to further improvements in the level of reproducibility and accuracy of HER2 testing and increase the proportion of patients accurately identified as eligible for anti-HER2 therapy.

\section{When to Test}

There is debate over when to assess HER2 status. Recent testing guidelines from ASCO (18) recommend evaluating HER2 status on every primary breast cancer either at diagnosis or at the time of recurrence. The German Pathology Advisory Board also supports early determination of HER2 status. Advocates of early testing cite the fact that HER2 positivity is an early event in breast cancer development and that the HER2 status of primary tumors appears to correlate with that of metastatic sites (20-25). However, others feel that it is only relevant to test for HER2 status in patients with advanced disease because this is the setting for which Herceptin is licensed. Of note, there is huge variation in national regulations on storing paraffin blocks: for example, although there is no legal requirement in Germany, pathologists in France and Canada are legally required to store blocks for 10 and 15 years, respectively. It is well known that breast cancer can recur up to 20 years after the first diagnosis. So, there is a practical argument for determining and recording the HER2 status of samples at diagnosis for use when the disease recurs.

Early testing for HER2 status could be particularly relevant if there is conclusive evidence of the value of HER2 in predicting response to adjuvant therapies. Current data suggest that HER2 positivity predicts response to anthracycline-based therapy (2629). Most reports also suggest that a HER2-positive status predicts resistance to hormonal therapy (30$34)$. One study suggests that the aromatase inhibitor letrozole may be more effective than tamoxifen in patients whose tumors are either EGFR (HER1) or HER2 positive and estrogen receptor (ER) positive (35). If these limited studies are confirmed by large, prospective, randomized trials, there will be strong pressure to screen all patients presenting with breast cancer for HER2 status. Studies are also investigating the clinical benefit of adjuvant Herceptin, which may ultimately necessitate HER2 testing at the time of diagnosis.

TABLE 1. Areas in Which National Guidelines Make Recommendations Regarding How to Test for HER2 Status

\begin{tabular}{|c|c|c|c|c|c|c|c|c|c|c|}
\hline & \multicolumn{10}{|c|}{ Recommendation Made By } \\
\hline & Australia (12) & Canada (13) & Czech Republic $^{\dagger}$ & Denmark $^{\dagger}$ & Finland $^{\dagger}$ & France (14) & Germany (15) & Japan (16) & Sweden $^{\dagger}$ & UK (17) \\
\hline When to test? & & & & & & & $\sqrt{ }$ & & & \\
\hline Technique & $\sqrt{ }$ & $\sqrt{ }$ & $\sqrt{ }$ & $\sqrt{ }$ & $\sqrt{ }$ & $\sqrt{ }$ & $\sqrt{ }$ & $\sqrt{ }$ & $\sqrt{ }$ & $\sqrt{ }$ \\
\hline $\begin{array}{l}\text { Guidelines for sample } \\
\text { preparation }\end{array}$ & & $\sqrt{ }$ & $\sqrt{ }$ & $\sqrt{ }$ & & & $\sqrt{ }$ & & $\sqrt{ }$ & $\sqrt{ }$ \\
\hline \multicolumn{11}{|l|}{ Antibody/probe } \\
\hline IHC & & $\checkmark$ & & $\sqrt{ }$ & $\checkmark$ & $\checkmark$ & $\sqrt{ }$ & $\sqrt{ }$ & $\sqrt{ }$ & $\sqrt{ }$ \\
\hline FISH & & $\sqrt{ }$ & & & & & $\sqrt{ }$ & & $\sqrt{ }$ & $\sqrt{ }$ \\
\hline \multicolumn{11}{|l|}{ Methodology } \\
\hline IHC & & $\sqrt{ }$ & & & & $\sqrt{ }$ & & & & $\sqrt{ }$ \\
\hline FISH & & & & & & $\sqrt{ }$ & & & & $\sqrt{ }$ \\
\hline Controls & $\sqrt{ }$ & $\sqrt{ }$ & & $\sqrt{ }$ & & $\sqrt{ }$ & & & $\sqrt{ }$ & $\sqrt{ }$ \\
\hline Scoring system & $\sqrt{ }$ & $\sqrt{ }$ & & $\sqrt{ }$ & & $\sqrt{ }$ & & $\sqrt{ }$ & & $\sqrt{ }$ \\
\hline What to report & & $\sqrt{ }$ & $\sqrt{ }$ & & & $\sqrt{ }$ & & & & \\
\hline Testing algorithm & $\sqrt{ }$ & $\sqrt{ }$ & $\sqrt{ }$ & & $\sqrt{ }$ & $\checkmark$ & $\sqrt{ }$ & $\sqrt{ }$ & $\sqrt{ }$ & $\sqrt{ }$ \\
\hline $\begin{array}{l}\text { Comment on central } \\
\text { vs. local laboratory }\end{array}$ & $\sqrt{ }$ & & $\sqrt{ }$ & & $\sqrt{ }$ & & & & & $\sqrt{ }$ \\
\hline
\end{tabular}

${ }^{\dagger}$ Unpublished HER2 testing guidelines currently used in routine clinical practice at a national level. 


\section{Assay Method}

There is no agreement on the best method for determining HER2 status. Assays such as immunohistochemistry (IHC) and enzyme-linked immunosorbent assay (ELISA) detect HER2 receptor overexpression. Fluorescence in situ hybridization (FISH), Southern blotting, chromogenic in situ hybridization (CISH), and polymerase chain reaction (PCR) measure the level of HER2 gene amplification. However, many of these assays are currently limited to research. The two most commonly used HER2 assays in clinical diagnostics, and recommended by all current national testing guidelines, are IHC and FISH. At present, neither CAP (19) nor ASCO (18) recommends the use of one over the other. CAP recommendations state that currently, "It is unclear whether FISH assays are superior to IHC, or whether FISH should be considered an adjunct or replacement." Of note, CISH has superseded FISH in Finland as the recommended method for determining HER2 gene amplification and is performed only at the two national reference laboratories by highly trained personnel. However, $\mathrm{CISH}$ is not currently recommended for routine diagnostic use outside Finland.

The two most widely used techniques, IHC and FISH, can be conducted using a variety of antibodies (IHC) or probes (FISH), either as part of a kit or alone. Only the Danish testing guidelines specifically recommend one particular assay, the IHCbased HercepTest (DAKO). Whichever assay is used, it should be standardized by following written protocols and procedures and be regularly validated, internally and externally, through the implementation of quality control (QC) and quality assurance (QA) measures.

\section{Tissue Processing}

The handling and processing of tissue samples before the HER2 assay can affect the results. Thus, there is a need to standardize the steps and procedures involved, including the following:

Type of specimen

Time from excision to fixation

Specimen slicing before fixation

Duration of fixation

Type of fixative

Storage of paraffin-embedded specimens/tissue sections and slide preparation

\section{Type of Specimen}

It was originally thought that fresh (frozen) tissue samples would give the most accurate results. However, this is not the case for IHC and FISH. Currently, most HER2 testing is done when metastatic disease is diagnosed, using specimens taken at first diagnosis of the primary cancer and therefore likely to be stored for long periods. At the moment, surgically excised samples from lumpectomies or mastectomies are preferred, although if these are not available core biopsies can be used. Cell blocks from fine needle aspiration biopsy (FNAB) may also be useful, particularly in the metastatic setting (36, 37).

\section{Time from Excision to Fixation}

Time to fixation is an important issue. Tissue specimens should be fixed as soon as possible after removal, preferably within 1 hour, using standardized procedures and strictly sticking to fixation times.

\section{Specimen Slicing before Fixation}

The thickness of tissue slices before fixation can affect HER2 assay results by delaying the penetration of the fixative. Therefore, care should be taken to ensure that specimens are fixed optimally by slicing at $0.5-1.0 \mathrm{~cm}$ and fixing immediately.

\section{Duration of Fixation}

Optimal fixation is essential to ensure that the HER2 protein is preserved and then correctly identified by IHC. Optimal fixation times vary between a minimum of 6 hours to a maximum of 48 hours (Rüschoff J, personal communication). Even tissue samples from core biopsies need a minimum period of fixation. Currently, there are no data on the reliability of quick fixation using a microwave in HER2 assays; therefore, this method is not recommended for routine clinical practice.

\section{Type of Fixative}

Many different fixatives are used, including phosphate-buffered formalin, alcohol-formalin fixatives, and Bouin's solution. It has been reported that the type of fixative can affect the assessment of HER2 by IHC and introduce artifacts $(38,39)$. For example, formalin fixation is associated with some loss of HER2 overexpression, as determined by IHC, especially if fixation is for $<24$ hours or $>48$ hours. Alcohol-based fixatives, such as Z-5 and Pen-Fix, can generate false-positive cases when using IHC (39). In France, Bouin's solution is commonly used although it has the disadvantage of making retrospective FISH testing impossible. Alcohol-based fixatives can also hamper FISH testing. Of the national testing guidelines that specifically recommend fixatives (Australian [12], Czech Republic [unpublished data], Canadian [13], and United Kingdom [17]), all suggest using $10 \%$ phosphate-buffered or neutral-buffered formalin.

\section{Storage of Paraffin-Embedded Specimens/Tissue Sections and Slide Preparation}

After fixation and processing, tissue specimens are usually embedded in paraffin. Properly fixed 
and paraffin-embedded samples for HER2 testing will keep indefinitely before sectioning if stored at room temperature $\left(20-25^{\circ} \mathrm{C}\right)$. There is anecdotal evidence that paraffin block sections should not be cut from the blocks and left at room temperature for a significant period of time before HER2 testing, as this may result in some loss of antigen in the section prepared. According to the recommendations from the manufacturer of the HercepTest, tissue sections mounted on slides and stored at room temperature $\left(20-25^{\circ} \mathrm{C}\right)$ should be stained within 4-6 weeks of sectioning to maintain antigenicity. For FISH testing, the United Kingdom guidelines suggest that storing cut sections for more than 6-12 months should be avoided.

The thickness of the tissue sections can affect the visualization and interpretation of assay results. Therefore sections should be cut at the standard thickness of 3-5 $\mu \mathrm{m}$ for IHC and $4-5 \mu \mathrm{m}$ for FISH. A hematoxylin and eosin (H\&E) section should be evaluated along with the IHC or FISH sections to ensure that there is adequate tumor tissue versus normal tissue and also to confirm the presence of an invasive component in the tumor.

\section{Immunohistochemistry}

\section{Methodology}

There are several factors that may contribute to the varying results observed with IHC assays:

Sensitivity and specificity of the antibodies

Use of antigen retrieval techniques

Antibody dilution

$\mathrm{pH}$ of buffer

Sensitivity and specificity of the detection system

Although there are a large number of anti-HER2 antibodies (each targeting different epitopes on the HER2 receptor), the most commonly used are the polyclonal antibody A0485, used alone or as part of the HercepTest, and the monoclonal antibodies CB11 (alone or as part as the Ventana PathWay kit) and TAB250. Several investigators have reported that the sensitivity and specificity of anti-HER2 monoclonal and polyclonal antibodies differ, although a number of these studies have also shown a high rate of concordance among the different anti-HER2 antibodies (39-44).

It is important to be aware of the specificity and sensitivity of individual antibodies and to take into account that antigen retrieval increases the sensitivity of the antibody at the expense of specificity. The manufacturer's guidelines for the HercepTest specify that wet antigen retrieval, for example involving a water bath, should be used. However, antigen retrieval with a microwave is sometimes used with other antibodies although it can be more difficult to standardize. Whichever method is cho- sen, it should be closely monitored and standardized and should follow strict protocols (45). The United Kingdom guidelines suggest monitoring the antigen retrieval process by using normal breast epithelium for comparison. If excess antigen retrieval has occurred and normal epithelium stains positive, then it is suggested that the assay should be rejected and the sample retested. The increased sensitivity associated with antigen retrieval can be balanced by diluting the antibody for more optimal results. The $\mathrm{pH}$ of the buffer can affect the concentration of the antibody (46). Therefore, antibody dilution should be optimized for each laboratory and re-examined every time new reagents are used. The dilution should be calibrated using tissue arrays or cell lines as controls.

Most of the national guidelines suggest using one of the commonly used antibodies for IHC (Table 2). Some national guidelines, such as the Canadian and Swedish, recommend using at least two IHC antibodies with complementary specificity and sensitivity for HER2 testing. Only the Danish testing guidelines stipulate using a particular IHC assay, the HercepTest. Although some groups have reported that the HercepTest has good correlation with FISH as long as the manufacturer's protocol is scrupulously followed $(47,48)$, others have noted that the HercepTest can be associated with a high degree of false-positive results $(38,41,49,50)$, particularly when the score is determined to be IHC $2+$. Thus, an internal validation of this kit is warranted before first use. Whichever antibody is used, the issues and pitfalls should be understood and considered when conducting the assay and interpreting results. It is essential to validate the assay first, and QC and QA measures should be undertaken regularly, internally and externally (Table 3).

\section{Quality Control}

The term quality control describes the internal validation procedures needed to guarantee the accuracy of a batch of HER2 test results. There should be standard operating procedures for IHC assays and the protocols should be calibrated, either by comparing results with those achieved using an-

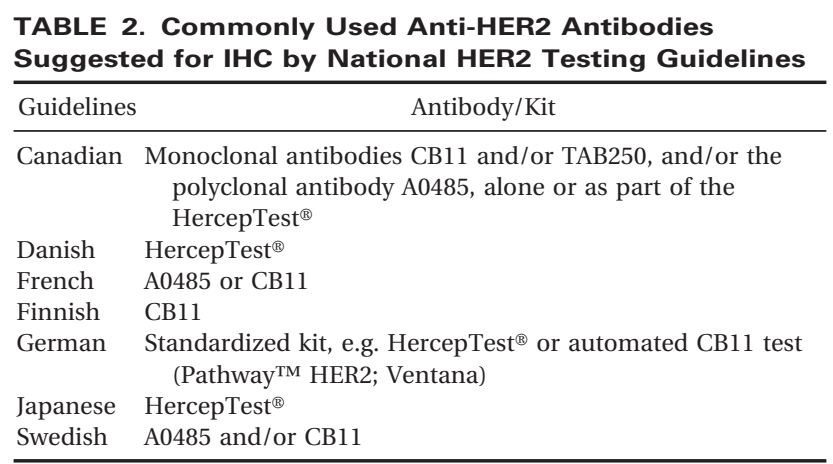


Internal quality controls

a) Staining:

b) Scoring:

c) Laboratory:

d) Testing process:

e) Documentation:

Continuous quality assessment

a) Provide training and education for staff

b) Repeat approximately $5 \%$ of positive and

negative cases per year

c) Participate in ring studies
Strict application of staining (IHC) or hybridization (FISH) protocols

Use of control slides: cell lines and tissue samples

for IHC of scores $0 / 1+, 2+, 3+$ proved by FISH

Repeat assay if control slides fail to meet acceptance criteria (according to

manufacturers' guidelines)

Evaluate:

IHC slides only if normal tissue is negative and positive staining is membranous

FISH slides with 60 evaluable nuclei and adequate quality (signal intensity,

background, no cross hybridization)

Invasive tumor only (!); in IHC $3+>50 \%$ of cells are usually positive

Inhomogeneous: evaluate best representative areas

Heterogeneous distinct clones: take the highest score

Internal control:

Frequency of IHC $2+/ 3+(15-25 \%)$

Concordance between IHC and FISH: $3+>90 \%, 2+<25 \%$

HER2 positive tumors are usually high grade, not lobular, and ER/PR negative

Equivocal cases 1) Repeat test

2) For IHC compare result with FISH

3) Consult external laboratory

Use standard result forms

Document any:

Discrepancies with general roles of specimen handling and processing

Difficulties in evaluating and interpreting slides other technology, such as FISH, or by comparing them with an external control, such as cell lines calibrated by IHC or FISH. The use of controls of known HER2 levels alongside the assay procedure is mandatory, and most of the national testing guidelines stipulate inclusion of positive and negative controls (determined by IHC and FISH) as a minimum. Additional controls close to cutoff values are also recommended. Controls can be tissue arrays, tissue specimens of known immunoreactivity, or cell lines (51). As an example, SK-BR-3 is a high HER2-overexpressing cell line, MDA-175 overexpresses HER2 at an intermediate level, and the cell lines MDA-231 and MCF-7 do not overexpress HER2 and so can act as negative controls.

\section{Quality Assurance}

Quality assurance is the technical evaluation that compares the results from one laboratory with those from other laboratories (i.e., external controls). HER2 testing laboratories in the United Kingdom, Canada, France, and Germany are encouraged to join external technical QA programs, which can include confirmation of the percentage of positive and negative IHC results from one laboratory by retesting samples with another assay. For example, the Canadian guidelines recommend that $5 \%$ of samples should be retested by IHC at a designated reference laboratory and that if there are any discrepancies, the sample should be retested by FISH. Other QA initiatives include sending samples to a reference laboratory for confirmation by FISH. Of note, the concordance between high HER2 overexpression (IHC $3+$ ), and FISH is around $90 \%$ (41, 52-57). However, the concordance between IHC at the 2+ level and FISH can be as low as $25 \%(41,54$, 57), and most guidelines recommend retesting all IHC $2+$ samples with FISH. Ring studies can also help promote training and experience among diagnostic personnel.

\section{Result Assessment}

Interpretation of stained samples can be subject to interobserver variability, which may affect the results $(44,58-60)$. Most national testing guidelines recommend the following when interpreting IHC results:

Score the percentage and intensity of cells showing complete membrane staining

Cytoplasmic staining should not be included when interpreting results

Assess staining in the invasive component but not in the in situ component

Normal epithelial cells should not stain. If staining is noted, the test should be rejected

Be aware of retraction artifacts, which may be falsely interpreted as positive

There are several methods of interpreting cells stained by IHC. The most common scoring system is recommended in the HercepTest manufacturer's protocol, but this has practical difficulties and is open to interpretation errors, particularly around the subjective IHC $2+/ 3+$ cutoff point. However, 
the staining intensity can be compared with controls included with the HercepTest kit as an interpretation guide. DAKO, the manufacturer of the HercepTest, have recently modified their recommendations and propose FISH testing of all IHC $2+$ tumors. The Finnish and Swedish guidelines advocate retesting all IHC $2+$ and $3+$ samples by in situ hybridization, diminishing the need to make a distinction between IHC $2+$ and $3+$ cases. However, this approach would be associated with increased cost implications. It should also be pointed out that the HercepTest scoring system may not be appropriate for use with other antibodies. Therefore, diagnostic personnel should conduct substantial validation studies to find the threshold of positivity for the system in their laboratory. Even so, there can still be a high level of interobserver variability, especially when distinguishing the $2+/ 3+$ or equivalent cutoff. The Canadian guidelines recommend a cutoff for positivity of $10 \%$ of cells with moderate/strong complete membrane staining. Using this, a high level of concordance has been noted between IHC (CB11) and PCR (61). We believe that if there is any doubt over a score of $3+$, it should be classified as equivocal and retested by another method, such as FISH.

Definitions of equivocal samples include the following:

Heterogeneous staining that may hinder interpretation

Cytoplasmic or retraction artifact obscuring the interpretation of true membrane staining

Weak staining in $>30 \%$ of tumor cells

Staining of normal epithelium

Extensive incomplete membrane staining

There are several ways of improving the interpretation of IHC scores. The Australian and French guidelines suggest a regular audit of HER2-positive results in an unselected breast cancer population to check that these are within the reported limits of $15-25 \%(62,63)$. There is also an association between certain histological types of tumor and HER2 status (64), and this can be used to question a HER2 result. The French and Canadian guidelines note that positivity of classic lobular carcinoma, mucinous and tubular carcinoma, or the absence of immunoreactivity in high-grade infiltrating ductal carcinomas should alert the pathologist to query the results. The Canadian guidelines also suggest questioning a negative HER2 result in high-grade, ER-negative clinically aggressive tumors or a negative result in Paget's disease or inflammatory carcinoma.

\section{Fluorescence In Situ Hybridization}

\section{Probes}

FISH has been shown to be a highly reproducible technique for HER2 testing, and prolonged storage of paraffin blocks does not appear to affect its sensitivity. Two FISH kits are commercially available: INFORM determines the absolute level of HER2 gene signals; PathVysion recognizes the potential for cells to be polysomic for chromosome 17 by calculating the ratio of HER2 gene to chromosome 17 centromere. It should be noted that the clinical significance of polysomy to anti-HER2 therapy is unknown. Both FISH assays take 2 days and require the use of expensive fluorescent microscopy. Most national testing guidelines favor the PathVysion assay. Personnel evaluating FISH results require extensive training and expertise to distinguish between normal and malignant cells and intraductal and invasive tumor cancer cells, and to spot artifacts.

\section{Quality Control and Quality Assessment}

QC and QA measures should be implemented for FISH assays, as discussed in the preceding section on IHC.

\section{Result Assessment}

FISH can quantify HER2 gene amplification. According to the manufacturers' protocols, HER2 gene amplification is either scored as an absolute value (INFORM; $>4$ considered positive) or as a ratio of HER2 gene amplification to chromosome 17 (PathVysion; $>2$ copies of HER2 for each chromosome 17 considered positive). However, these cutoff values are arbitrary and may well be revised in the future. Interpretation of FISH results can be difficult when the ratio is between 1.8-2.2 for the PathVysion kit or when there is a score of 4-6 with the INFORM kit. The current PathVysion scoring system was, however, clinically relevant when Herceptin pivotal trial data were re-analyzed and HER2 amplification was correlated with clinical outcome $(9,10)$.

The number of cells needed to determine the level of HER2 gene amplification varies in the literature from 20-100 (65). However, the manufacturers' protocols recommend that for HER2 gene amplification, 60 nuclei should be counted and an average score taken. Cells from different areas of the sample should be counted. Diagnostic personnel must have enough experience to ensure that the cells counted are invasive cancer cells. With the PathVysion kit only, cells with one or more FISH signals of each color should be scored. The final result should then be calculated as a ratio of average HER2 signal to that of chromosome 17 signal in 60 interphase tumor cells. A further issue with FISH is that excessive digestion can occur if the sample has been poorly fixed. Modifying the digestion time can give better visualization of the signal. One important point to note is that FISH is often considered to be the gold standard to which other HER2 
assays are compared. However, the interobserver variability with FISH has not been determined and requires further examination.

\section{What to Report}

There is no consensus of national testing guidelines on what should be reported. The guidelines from Canada and the Czech Republic advocate full reporting of methodology (including controls, kit or antibody/probe) and scoring method. This is in line with recent recommendations from ASCO (18) and CAP (19). The ASCO recommendations state that because different laboratories use different assays, reporting should include "not only an estimate of HER2 levels but also a statement about the test's quality controls, the method, the specific kit or reagent, details of the scoring system and a statement regarding reproducibility, sensitivity and specificity of the assay, and a reference to the clinical validation of the assay or its correlation with a clinically validated c-erbB-2 test." Such reporting may seem overly detailed, but it will help to improve the reproducibility of assays and decrease variability of results. When correlating results from different laboratories it may be important to know more than whether a HER2 result is positive or negative. As a minimum we recommend that the following should be reported:

Assay method

Antibody or probe

Scoring system (including number of cells analyzed for FISH)

Controls

Final score interpretation

\section{Where to Test}

Where to test is a contentious issue. Many of the national testing guidelines advocate centralized testing, at least to confirm equivocal IHC results. Centralized HER2 testing can be particularly useful for FISH, which needs extensive training and costly equipment. Centralized facilities assay greater numbers of samples per year, leading to greater experience and accuracy. Rigid quality control and validation add to the level of accuracy. But samples are generally fixed before being sent to the centralized facility and this cannot be controlled or standardized centrally. Local testing can be as accurate as centralized testing as long as there are education and training programs, standard and validated protocols, and quality control and assurance (48). Local laboratories can also provide results more quickly, with close contact between the pathologist and medical oncologist. However, a laboratory needs to test an adequate number of samples a year in order to have the technical experience to pro- duce optimal results. The NSABP has specified that a laboratory must test at least 100 samples a month before patients tested at the laboratory can enter the NSABP B-31 Herceptin adjuvant trial (66). It is recommended in the United Kingdom (though not stated in the current guidelines) that laboratories should ideally test $\geq 250$ samples a year by IHC (48).

\section{Suggested Algorithm}

HER2 positivity predicts a response to the antiHER2 monoclonal antibody therapy Herceptin. Patients with strong HER2 overexpression (IHC $3+$ ) or HER2 gene amplification benefit most from this therapy (7, 9-11). Testing algorithms should take this into consideration.

Most national testing guidelines suggest a similar testing algorithm. Tumor samples are initially tested by IHC. Samples with strong HER2 overexpression (IHC $3+$ ) indicate eligibility for Herceptin therapy. IHC $2+$ samples should be retested with another method, preferably FISH, to confirm results (Fig. 1). If FISH is used to determine HER2 status, amplification indicates eligibility for Herceptin therapy.

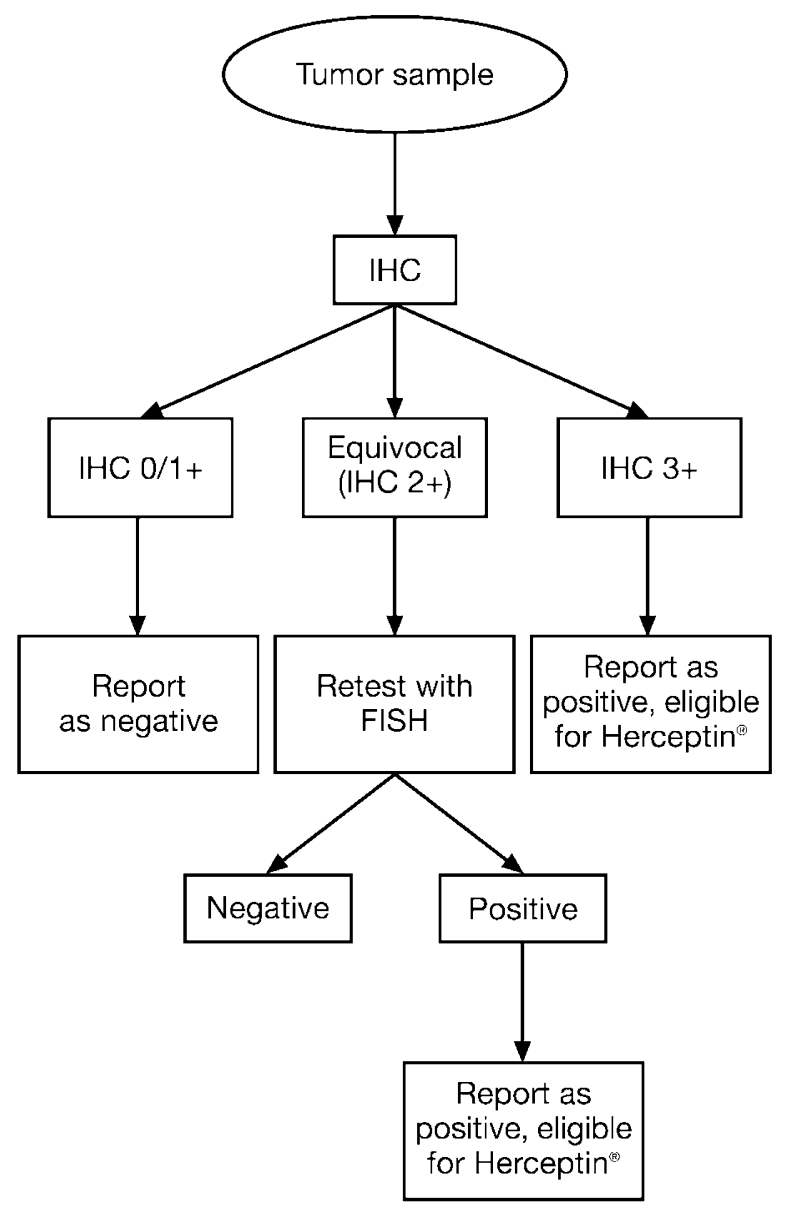

FIGURE 1. HER2 testing algorithm. 


\section{SUMMARY}

The consistent take-home message from national testing guidelines is the need to standardize HER2 testing procedures and to validate tests against a reference to improve accuracy. Protocols should be defined, reproducible, and strictly adhered to. There should be mandatory controls, and accuracy and reliability checks should be part of routine clinical practice. These procedures plus continued education and training are essential to accurately identify patients with HER2-positive breast cancer.

We recommend IHC as the screening test of choice for HER2 status, with the caveat that pathologists and laboratory personnel should receive continuous education in all aspects of this assay. It is clear that to reach a satisfactory level of standardization and accuracy, laboratories must do a reasonable number of assays a year to ensure quality of results. If a commercial IHC kit is used then every detail of the manufacturer's protocol should be scrupulously followed, particularly with antigen retrieval. If an in-house IHC assay is used, it should be validated against another technology (FISH or PCR) and controlled frequently. Ambiguous cases should be retested by another method, preferably FISH.

\section{REFERENCES}

1. Slamon DJ, Clark GM, Wong SG, Levin WJ, Ullrich A, McGuire WL. Human breast cancer: correlation of relapse and survival with amplification of the HER-2/neu oncogene. Science 1987;235:177-82.

2. Slamon D, Godolphin W, Jones LA, Holt JA, Wong SG, Keith DE, et al. Studies of the HER-2/neu protooncogene in human breast and ovarian cancer. Science 1989;244:707-12.

3. Ross JS, Fletcher JA. The HER-2/neu oncogene in breast cancer: prognostic factor, predictive factor, and target for therapy. Stem Cells 1998;16:413-28.

4. Dowsett M, Cooke T, Ellis I, Gullick WJ, Gusterson B, Mallon E, et al. Assessment of HER2 status in breast cancer: why, when and how? Eur J Cancer 2000;36:170-6.

5. Piccart M, Lohrisch C, Di Leo A, Larismont D. The predictive value of HER2 in breast cancer. Oncology 2001;61(Suppl 2):73-82.

6. Cobleigh MA, Vogel CL, Tripathy D, Robert NJ, Scholl S, Fehrenbacher L, et al. Multinational study of the efficacy and safety of humanized anti-HER2 monoclonal antibody in women who have HER2-overexpressing metastatic breast cancer that has progressed after chemotherapy for metastatic disease. J Clin Oncol 1999;17:2639-48.

7. Slamon DJ, Leyland-Jones B, Shak S, Fuchs H, Paton V, Bajamonde A, et al. Use of chemotherapy plus a monoclonal antibody against HER2 for metastatic breast cancer that overexpresses HER2. N Engl J Med 2001;344:783-92.

8. Vogel CL, Cobleigh MA, Tripathy D, Gutheil JC, Harris LN, Fehrenbacher L, et al. Efficacy and safety of trastuzumab as a single agent in first-line treatment of HER2-overexpressing metastatic breast cancer. J Clin Oncol 2002;20:719-26.

9. Mass RD, Press M, Anderson S, Murphy M, Slamon D. Improved survival benefit from Herceptin (trastuzumab) in patients selected by fluorescence in situ hybridization (FISH) [abstract]. Proc Am Soc Clin Oncol 2001;20:22a.
10. Vogel CL, Cobleigh D, Tripathy D, Mass R, Murphy M, Stewart SJ. Superior outcomes with Herceptin (trastuzumab) (H) in fluororescence in situ hybridization (FISH)-selected patients [abstract]. Proc Am Soc Clin Oncol 2001;20:22a.

11. Smith IE. Efficacy and safety of Herceptin in women with metastatic breast cancer: results from pivotal clinical studies. Anticancer Drugs 2001;12(Suppl 4):S3-10.

12. Bilous M. HER2 testing recommendations in Australia. Pathology 2001;33:425-7.

13. Hanna W, O'Malley FO. Updated recommendations from the HER2 / neu consensus meeting-Toronto, Ontario, 7 September, 2001. Curr Oncol 2002;9:S18-20.

14. Penault-Llorca F, Balaton A, Sabourin JC, Le Doussal V. Immunohistochemical evaluation of HER2 status in infiltrating breast cancers: development of technical protocol and reading of results-recommendations. Ann Pathol 2002;22: 150-7.

15. Schaller G. Metastasiertes mammakarzinom: welche patientinnen profitieren von einer antikörper-therapie? Dtsch Arzteblatt 2001;98:A3014-5.

16. Umemura S, Sakamoto G, Sasano H, Tsuda H, Akiyama F, Kurosumi M, et al. Evaluation of HER2 status: for the treatment of metastatic breast cancers by humanized anti-HER2 monoclonal antibody (trastuzumab) (Pathological Committee for Optimal use of Trastuzumab). Breast Cancer 2001;8: $316-20$.

17. Ellis IO, Dowsett M, Bartlett J, Walker R, Cooke T, Gullick W, et al. Recommendations for HER2 testing in the UK. J Clin Pathol 2000;53:890-2.

18. Bast RC, Ravdin P, Hayes DF, Bates S, Fritsche H Jr, Jessup $\mathrm{JM}$, et al. 2000 update of recommendations for the use of tumor markers in breast and colorectal cancer: clinical practice guidelines of the American Society of Clinical Oncology. J Clin Oncol 2001;19:1865-78.

19. Fitzgibbons PL, Page DL, Weaver D, Thor AD, Allred DC, Clark GM, et al. Prognostic factors in breast cancer: College of American Pathologists Consensus Statement 1999. Arch Pathol Lab Med 2000;124:966-78.

20. Cardoso F, Di Leo A, Larsimont D, Gancberg D, Rouas G, Dolci S, et al. Evaluation of HER2, p53, bcl-2, topoisomerase II-alpha, heat shock proteins 27 and 70 in primary breast cancer and metastatic ipsilateral axillary lymph nodes. Ann Oncol 2001;12:615-20.

21. Gancberg M, Di Leo A, Rouas G, Pedrocchi M, Paesmans M, Verhast A. Comparison of Topo-isomerase II $\alpha$ (topoII $\alpha$ ) and HER-2 status between primary (P) breast cancer (BC) and corresponding metastatic (M) sites [abstract]. Proc Am Assoc Cancer Res 2001;42:8.

22. Larsimont D, Di Leo A, Rouas G, Pedrocchi M, Paesmans M, Verhast A, et al. Primary versus metastatic breast cancer. A comparison of HER-2 and Topo-Isomerase II $\alpha$ status [abstract]. Proc Am Soc Clin Oncol 2001;20:426a.

23. Lower EE, Yassin R, Blau R, Heffelfingers S. HER-2/neu expression in primary breast cancer correlates with metastatic disease expression [abstract]. Proc Am Soc Clin Oncol 2001; 20:425a.

24. Simon R, Nocito A, Hubscher T, Bucher C, Torhurst J, Schraml P, et al. Patterns of HER-2/neu amplification and overexpression in primary and metastatic breast cancer. J Natl Cancer Inst 2001;93:1141-6.

25. Tanner M, Järvinen $\mathrm{P}$, Isola J. Amplification of HER-2/neu and topoisomerase II $\alpha$ in primary and metastatic breast cancer. Cancer Res 2001;61:5345-8.

26. De Laurentiis M, Caputo F, Massarelli E, Forestieri V, Vernaglia A, Carlomagno C, et al. HER2 expression and anthracycline effect: results from the Naples GUN3 randomised trial [abstract]. Proc Am Soc Clin Oncol 2001;20:34a.

27. Paik S, Bryant J, Tan-Chiu E, Yothers G, Park C, Wickerham DL, et al. HER2 and choice of adjuvant chemotherapy for 
invasive breast cancer: National Surgical Adjuvant Breast and Bowel Project protocol B-15. J Natl Cancer Inst 2000;92: 1991-8.

28. Ravdin PM, Green S, Albain V, Boucher V, Ingle J, Pritchard $\mathrm{K}$, et al. Initial report of the SWOG biological correlative study of c-erbB-2 expression as a predictor of outcome in a trial comparing adjuvant CAF with tamoxifen (T) alone [abstract]. Proc Am Soc Clin Oncol 1998;17:97a.

29. Thor AD, Berry DA, Budmann DR, Muss HB, Kute T, Henderson IC, et al. ErbB-2, p53, and efficacy of adjuvant therapy in lymph node-positive breast cancer. J Natl Cancer Inst 1998;90:1346-60.

30. Bianco AR, De Laurentiis M, Carlomagno C, Gallo C, Panico L, De Placido S. HER2 overexpression predicts adjuvant tamoxifen (TAM) failure for elderly breast cancer (EBC): complete data at $20 \mathrm{yr}$ of the Naples GUN randomized trial [abstract]. Proc Am Soc Clin Oncol 2000;19:75a.

31. Colomer R, Llombart A, Ramos M, Mayordomo JI, Fernandez $\mathrm{R}$, Tusquets I, et al. Serum HER-2 ECD and the efficacy of letrozole in $\mathrm{ER}+/ \mathrm{PR}+$ metastatic breast cancer: preliminary results of a prospective study [abstract]. Breast Cancer Res Treat 2001;69:242.

32. Giai M, Roagna R, Ponzone R, De Bortoli M, Dati C, Sismondi P. Prognostic and predictive relevance of c-erbB-2 and ras expression in node positive and negative breast cancer. Anticancer Res 1994;14:1441-50.

33. Lipton A, Ali SM, Leitzel K, Demers L, Chinchilli V, Engle L, et al. Elevated serum HER-2/neu level predicts decreased response to hormone therapy in metastatic breast cancer. J Clin Oncol 2002;20:1467-72.

34. Stål O, Borg A, Ferno M, Kallstrom AC, Malmstrom P, Nordenskjold B. ErbB2 status and the benefit from two or five years of adjuvant tamoxifen in postmenopausal early stage breast cancer. Ann Oncol 2000;11:1545-50.

35. Ellis MJ, Coop A, Singh B, Mauriac L, Llombert-Cussac A, Janicke $\mathrm{F}$, et al. Letrozole is more effective neoadjuvant endocrine therapy than tamoxifen for ErbB-1- and/or ErbB2-positive, estrogen receptor-positive primary breast cancer: evidence from a phase III randomized trial. J Clin Oncol 2001;19:3808-16.

36. McManus DT, Patterson AH, Maxwell P, Humphreys MW, Anderson NH. Fluorescence in situ hybridisation detection of erbB2 amplification in breast cancer fine needle aspirates. Mol Pathol 1999;52:75-7.

37. Mezzelani A, Alasio L, Bartoli C, Bonora MG, Pierotti MA, Rilke F. c-erbB2/neu gene and chromosome 17 analysis in breast cancer by FISH on archival cytological fine-needle aspirates. Br J Cancer 1999;80:519-25.

38. Jacobs TW, Gown AM, Yaziji H, Barnes MJ, Schnitt SJ. Specificity of HercepTest in determining HER-2/neu status in breast cancer using the United States Food and Drug Administration-approved scoring system. J Clin Oncol 1999; 17:1983-7.

39. Penault-Llorca F, Adelaide J, Houvenaeghel G, Hassoun J, Birnbaum D, Jacquemier J. Optimization of immunohistochemical detection of ERBB2 in human breast cancer: impact of fixation. J Pathol 1994;173:65-75.

40. Gancberg D, Lespagnard L, Rouas G, Paesmans M, Piccart M, Di Leo A, et al. Sensitivity of HER-2/neu antibodies in archival tissue samples of invasive breast carcinomas. Correlation with oncogene amplification in 160 cases. Am J Clin Pathol 2000;113:675-82.

41. Lebeau A, Deimling D, Kaltz C, Sendelhofert A, Iff A, Luthardt B, et al. Her-2/neu analysis in archival tissue samples of human breast cancer: comparison of immunohistochemistry and fluorescence in situ hybridization. J Clin Oncol 2001;19:354-63.

42. O'Malley FP, Parkes R, Latta E, Tjan S, Zadro T, Mueller R, et al. Comparison of HER2/neu status assessed by quantitative polymerase chain reaction and immunohistochemistry. Am J Clin Pathol 2001;115:504-11.

43. Press MF, Hung G, Godolphin W, Slamon DJ. Sensitivity of HER-2/neu antibodies in archival tissue samples: potential source of error in immunohistochemical studies of oncogene expression. Cancer Res 1994;54:2771-7.

44. Thomson TA, Hayes MM, Spinelli JJ, Hiland E, Sawrenko C, Phillip D, et al. HER-2/neu in breast cancer: interobserver variability and performance of immunohistochemistry with 4 antibodies compared with fluorescent in situ hybridization. Mod Pathol 2001;14:1079-86.

45. Farabegoli F, Ceccarelli C, Santini D, Baldini N, Taffurelli M, Marrano D, et al. c-erbB-2 over-expression in amplified and non-amplified breast carcinoma samples. Int J Cancer 1999; 84:273-7.

46. Shi SR, Imam SA, Young L, Cote RJ, Taylor CR. Antigen retrieval immunohistochemistry under the influence of $\mathrm{pH}$ using monoclonal antibodies. J Histochem Cytochem 1995; 43:193-201.

47. Birner P, Oberhuber G, Stani J, Reithofer C, Samonigg H, Hausmaninger $\mathrm{H}$, et al. Evaluation of the United States Food and Drug Administration-approved scoring and test system of HER-2 protein expression in breast cancer. Clin Cancer Res 2001;7:1669-75.

48. Di Leo A, Dowsett M, Horten B, Penault-Llorca F. Current status of HER2 testing. Oncology 2002;63(Suppl 1):25-32.

49. Roche PC, Ingle JN. Increased HER2 with U.S. Food and Drug Administration-approved antibody. J Clin Oncol 1999; 17:434.

50. Tubbs RR, Pettay JD, Roche PC, Stoler MH, Jenkins RB, Grogan TM. Discrepancies in clinical laboratory testing of eligibility for trastuzumab therapy: apparent immunohistochemical false-positives do not get the message. J Clin Oncol 2001;19:2714-21.

51. Rhodes A, Jasani B, Couturier J, McKinley MJ, Morgan JM, Dodson AR, et al. A formalin-fixed, paraffin-processed cell line standard for quality control of immunohistochemical assay of HER-2/neu expression in breast cancer. Am J Clin Pathol 2002;117:81-9.

52. Hoang MP, Sahin AA, Ordòñez NG, Sneige N. HER-2/neu gene amplification compared with HER-2/ neu protein overexpression and interobserver reproducibility in invasive breast carcinoma. Am J Clin Pathol 2000;113:852-9.

53. Jacobs TW, Gown AM, Yaziji H, Barnes MJ, Schnitt SJ. Comparison of fluorescence in situ hybridization and immunohistochemistry for the evaluation of HER-2/neu in breast cancer. J Clin Oncol 1999;17:1974-82.

54. Jimenez RE, Wallis T, Tabasczka P, Visscher DW. Determination of Her-2/ neu status in breast carcinoma: comparative analysis of immunohistochemistry and fluorescent in situ hybridization. Mod Pathol 2000;13:37-45.

55. Onody P, Bertrand F, Muzeau F, Bieche I, Lidereau R. Fluorescence in situ hybridization and immunohistochemical assays for HER-2/neu status determination: application to node-negative breast cancer. Arch Pathol Lab Med 2001;125: $746-50$.

56. Ridolfi RL, Jamehdor MR, Arber JM. HER-2/neu testing in breast carcinoma: a combined immunohistochemical and fluorescence in situ hybridization approach. Mod Pathol 2000;13:866-73.

57. Wang S, Saboorian MH, Frenkel E, Hynan L, Gokaslan ST, Ashfaq R. Laboratory assessment of the status of Her-2/neu protein and oncogene in breast cancer specimens: comparison of immunohistochemistry assay with fluorescence in situ hybridisation assays. J Clin Pathol 2000;53:374-81.

58. Bloom K, de la Torre-Bueno J, Press M, Gown A, Bauer K, Harrington D. Comparison of HER-2/neu analysis using FISH and IHC when HercepTest is scored using conven- 
tional microscopy and image analysis [abstract]. Breast Cancer Res Treat 2000;64:99.

59. Kay EW, Walsh CJ, Cassidy M, Curran B, Leader M. C-erbB-2 immunostaining: problems with interpretation. J Clin Pathol 1994;47:816-22.

60. Torre-Bueno J, Harrington DS, Bauer KD. Use of image analysis to improve accuracy of reading HER2Neu status by IHC [abstract]. Proc Am Assoc Cancer Res 2001;42:118.

61. Hanna WM, Kahn HJ, Pienkowska M, Blondal J, Seth A, Marks A. Defining a test for HER-2/neu evaluation in breast cancer in the diagnostic setting. Mod Pathol 2001;14:677-85.

62. Hynes NE, Stern DF. The biology of erbB-2/neu/HER-2 and its role in cancer. Biochem Biophys Acta Rev Cancer 1994; 1198:165-84.
63. Révillion F, Bonneterre J, Peyrat JP. ERBB2 oncogene in human breast cancer and its clinical significance. Eur J Cancer 1998;34:791-808.

64. Bilous M. Predicting HER2 status of breast cancer from basic pathology features: HER2 status of 1500 breast cancers determined by immunohistochemistry and fluorescence in situ hybridisation with pathology correlation [abstract]. Eur J Cancer 2002;38:S52.

65. Persons DL, Bui MM, Lowery MC, Mark HF, Yung JF, Birkmeier JM, et al. Fluorescence in situ hybridization (FISH) for the detection of HER-2/neu amplification in breast cancer: a multicenter portability study. Ann Clin Lab Sci 2000;30:41-8.

66. Tan-Chiu E, Piccart M. Moving forward: Herceptin in the adjuvant setting. Oncology 2002;63(Suppl 1):57-63. 